\title{
Low voltage $I-V$ characteristics in magnetic tunneling junctions
}

\author{
G. G. Cabrera ${ }^{a)}$ \\ Instituto de Física Gleb Wataghin, Universidade Estadual de Campinas (UNICAMP), C.P. 6165, \\ Campinas 13083-970 SP, Brazil \\ N. García \\ Laboratorio de Física de Sistemas Pequeños y Nanotecnología, Consejo Superior de Investigaciones \\ Científicas (CSIC), Serrano 144, E-28006 Madrid, Spain
}

(Received 5 July 2001; accepted for publication 5 November 2001)

\begin{abstract}
We show that elastic currents, which take into account variations of the tunneling transmission with voltage and a large ratio of majority to minority spin densities of states of the conduction band at the Fermi level, can account for the low voltage current anomalies observed in magnet-oxidemagnet junctions. (C) 2002 American Institute of Physics. [DOI: 10.1063/1.1433168]
\end{abstract}

In 1975 , Julliere ${ }^{1}$ proposed a simple model for tunneling between two ferromagnetic metals, assuming that the spin is conserved in the tunneling process and that the tunneling current is proportional to the density of states of each spin at the ferromagnetic electrodes. A magnetoresistance (MR) effect then appears when one compares the resistance for cases in which the magnetization of the electrodes is antiparallel (AP) and parallel (P). However, it is only quite recently that tunneling ferromagnetic samples have been shown to produce the large magnetoresistance effect $(25 \%-30 \%)$ found, for example, in Refs. 2-4. In addition, a remarkable dependence of the junction conductance on the voltage bias $(V)$ has been observed at low voltages (of the order of a few hundred millivolts). In experiments reported in Refs. 3 and 4, the junction resistance drops significantly with applied voltage, with a peak at zero bias (called the zero-bias anomaly) that is more pronounced for AP alignment. The effect is also temperature dependent, with the peak being less sharp at room temperature. Finally, it is found that the junction magnetoresistance (JMR) has a large decrease with the voltage, up to $60 \%$ at $0.5 \mathrm{~V}$ in some cases. ${ }^{4}$ Early theoretical calculations of tunneling currents with applied voltages by Simmons ${ }^{5}$ showed variations of the conductance that are of the order of that observed in Refs. 3 and 4, but his theory yields no structure at zero bias.

Scattering from surface magnons has been proposed as a mechanism for randomizing the tunneling process and opening the spin-flip channels that reduce the MR. ${ }^{3}$ While the above phenomenon may explain the JMR behavior in the immediate vicinity of the zero-bias peak (for voltages smaller than $\sim 40 \mathrm{mV}$ ), estimations of magnon scattering cross sections show that the effect is too small to account for the sharp drop in resistance in the whole range of 400-500 mV observed in Refs. 3 and 4. In fact, inelasticelectron tunneling spectroscopy (IETS) measurements at low temperature $^{4,6}$ showed peaks which can unambiguously be associated with one-magnon spectra at very small voltages (from 12 to $20 \mathrm{mV}$, with tails up to $40 \mathrm{mV}$ ). Also, early experiments with spin polarized photoelectrons that tunnel from a ferromagnet through the surface barrier indicate that

${ }^{a)}$ Electronic mail: cabrera@ifi.unicamp.br inelastic electron-magnon scattering at the interfaces can be neglected and that the spin is conserved in the tunneling process (no contribution by spin flip to the photoemission current). ${ }^{7}$ In any case, the estimation of the matrix elements for one-magnon processes in tunneling barriers is very difficult, while its magnitude is treated as an adjustable parameter in Ref. 3. Even if one takes this parameter at face value, the calculation developed in Ref. 3 uses perturbation theory for one-magnon exchange, and should be valid for voltages smaller than the maximum magnon frequency, which is typically of the order of $100 \mathrm{meV}$. To extend the theory beyond this limit will imply the inclusion of multimagnon processes, which are negligible at low temperature. Contrary to the claim made in Ref. 3 , in the present letter we show that the experimental data can be explained by elastic tunneling currents.

We propose an extension of Simmons' tunneling theory ${ }^{5}$ that includes band structure effects on voltage-dependent elastic currents that conserve spin. The theory takes into account variations of the density of states with the bias at both magnetic electrodes. This is relevant, since experiments probe depths of the order of $0.5 \mathrm{eV}$ from the Fermi surface. Several possibilities can be introduced, depending on the position of the Fermi level in the spin-split band picture of ferromagnetic metals. ${ }^{8}$

This latter consideration brings into question the nature of spin polarization in transition ferromagnets. In $3 d$ ferromagnets, most of the spin polarization comes from the $d$ band, whose density of state is typically 10 times bigger than that of the $s$ electrons, which in turn is poorly polarized. ${ }^{9}$ On the other hand, tunneling currents are dominated by $s$ band contributions. This is so, because $d$ wave functions are more localized and their effective tunneling barrier is higher. The inclusion of $s-d$ hybridization does not change this result significantly. ${ }^{10,11}$ For $\mathrm{Ni}$, it has been estimated that the tunneling probability of the $s$ electrons is of the order of 100 times that of the $d$ electrons, thus leading to positive spin polarization in Ni field emission experiments. ${ }^{12}$ As a net result, the contribution of the $d$ density of states is greatly reduced in tunneling phenomena. Within this context, we formulate a simple model with effective smooth bands for majority and minority spins using parabolic dispersions. Ap- 
preciable variations of the density of states are attained near the top or bottom of the bands. We obtain different behaviors for the zero-bias anomaly, that is, whether the Fermi level is located near the bottom (peak) or top of the bands (dip), or when the position of the Fermi level is close to the top of the majority band and the bottom of the minority one. They are possible scenarios that we may encounter in our model.

In order to develop our calculation, one has to rewrite Simmons' formulas with the conductance current written in the form

$$
\begin{aligned}
J^{(C)}(V)= & A \sum_{(\sigma, \mu) \in C} \int_{E_{F}}^{E_{F}+e V} d E T(E, \Delta s, \phi, V) \\
& \times N_{\mathrm{L}}^{(\sigma)}(E) N_{\mathrm{R}}^{(\mu)}(E+e V),
\end{aligned}
$$

where $A$ is a constant related to the charge $e$ of the carriers, $T(E, \Delta s, \phi, V)$ is the spin independent tunneling transmission through the barrier for energy $E$, parametrized with the mean barrier height $\phi$ and width $\Delta s,{ }^{5}$ the index $C=P, A P$ refers to the magnetic configuration (parallel or antiparallel), and $N_{\mathrm{L}, \mathrm{R}}$ are the local densities of states for the left and right electrodes, respectively, in the regions close to the insulator. The indexes $(\sigma, \mu)$ for the densities of states stand for $m a$ jority $(M)$ and minority $(m)$ spin bands, and a sum in expression (1) is made over the allowed processes for spin conserving tunneling, when the setup is in the $C$ magnetic configuration. We have assumed that both electrodes are made of the same ferromagnetic metal, with a symmetric conductance for positive and negative bias. For not too high temperatures, the Fermi distributions were approximated by step functions.

If the voltage is small compared to the Fermi energy, the tunneling current, as given by expression (1), approximately factorizes in the form of

$$
\begin{aligned}
J^{(C)}(V) & \approx\left(\sum_{\sigma, \mu=m, M}^{C} N^{(\sigma)}\left(E_{F}\right) N^{(\mu)}\left(E_{F}+e V\right)\right) J^{(S)}(V) \\
& =D^{(C)}\left(E_{F}, V\right) J^{(S)}(V),
\end{aligned}
$$

where $J^{(S)}(V)$ is the Simmons' tunneling current as a function of the voltage bias. ${ }^{5} J^{(S)}(V)$ is spin independent and carries all information concerning details of the tunneling barrier. The structure at zero bias is produced by the term $D^{(C)}\left(E_{F}, V\right)$ coming from the spin polarization at the Fermi level. This approximate factorization will imply some universal properties in the MR. More on this later.

We denote by $E_{M}$ and $E_{m}$ the bottom (top) of the majority and minority bands, respectively. In formulating the Stoner model within naive band theory, $\left|E_{m}-E_{M}\right|$ should yield the exchange of the band. But Fermi surfaces of transition metals are very intricate, having contributions from electron- and hole-like carriers and different shapes for majority and minority spin sheets. The exchange splitting is not rigid across the Brillouin zone and $s-d$ hybridization complicates this picture even more. ${ }^{9}$ In this context, $E_{m}$ and $E_{M}$ come from the band structure and $\Delta E=\left|E_{m}-E_{M}\right|$ may be very different from the true exchange of the band.

To parametrize our results, and denoting by $E_{F}$ the Fermi energy, we define

$$
E_{F}^{M} \equiv\left|E_{F}-E_{M}\right|, \quad E_{F}^{m} \equiv\left|E_{F}-E_{m}\right|, \quad E_{F}^{M} \equiv \lambda \quad E_{F}^{m},
$$

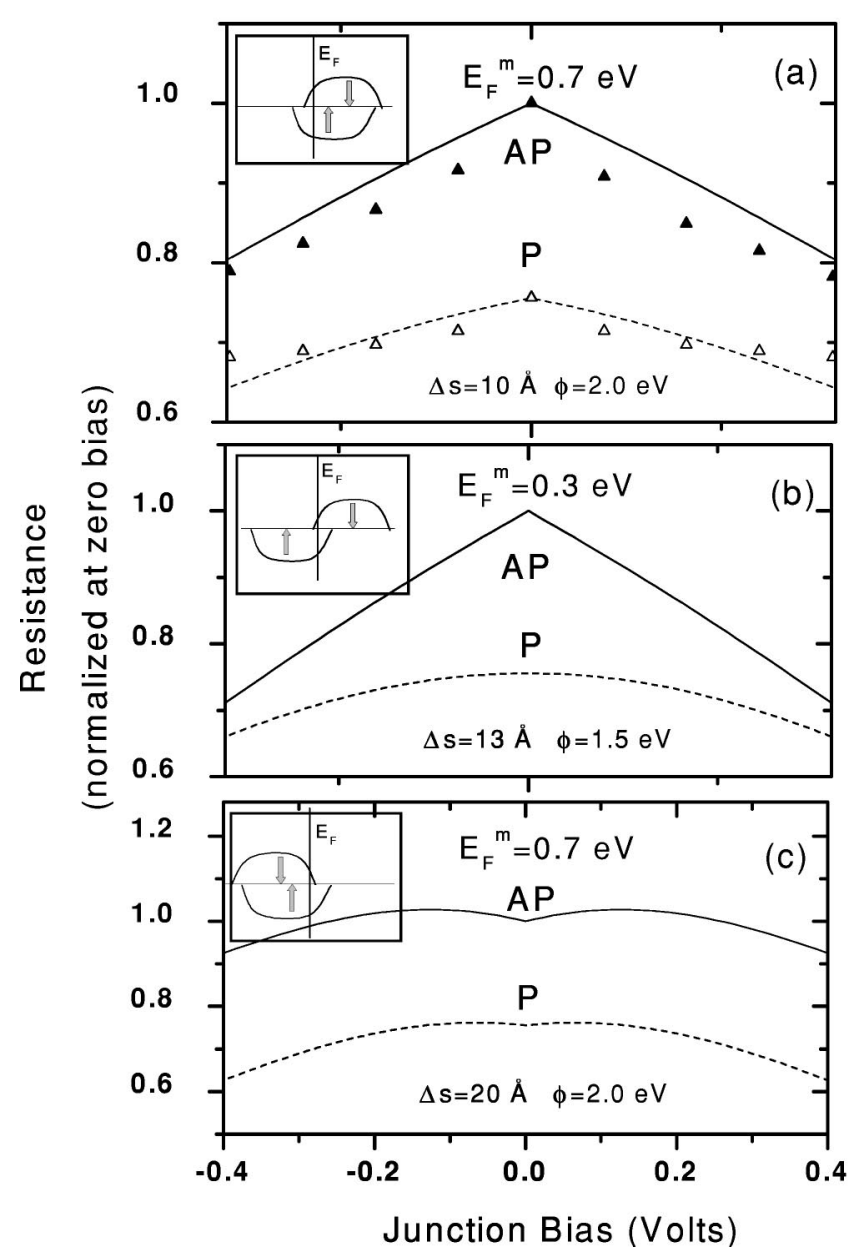

FIG. 1. Resistance as a function of the voltage bias for the AP and P configurations and for different band structures (shown in the insets). The parameters for the tunneling barriers are given. As a reference, representative experimental data points taken from Ref. 3 are shown in (a), where good agreement with our calculation is obtained with $\sqrt{\lambda} \approx 2.2$, the value used in the following. (a) electron-like carriers for both majority and minority spin bands; (b) hole-like majority and electron-like minority bands; (c) hole-like bands for both spins.

which includes both cases, the bottom and the top of the band. The ratio of the densities of states at the Fermi level is given by $N_{L}^{(M)}\left(E_{F}\right) / N_{L}^{(m)}\left(E_{F}\right)=\sqrt{\lambda}$. In Fig. 1, we display results of our calculation for examples of typical barriers. We have not tried an optimum fitting with experiments, because this depends on details of the tunneling junction which, in general, are not given in the literature (effective barrier height and width: when absolute resistance is given, one can estimate the barrier height if the barrier width and the section of the junction are known ${ }^{11}$ ), but it is clear that the experimental results can only be explained by assuming large polarization of the band at the Fermi level. Note that polarization at the Fermi level may be inverted with respect to polarization of the whole band, as for instance in Fig. 1(c).

The change in tunnel resistance or magnetoresistance (MR) is defined by $\Delta R / R=\left(R_{\mathrm{AP}}-R_{\mathrm{P}}\right) / R_{\mathrm{AP}}$, and some results are displayed in Fig. 2. In Fig. 2(b), we show a comparison to different experimental results found in the literature. ${ }^{13}$

When the Fermi level lies near the top of the bands [Fig. 1(c)], there is an increase in MR with voltage bias. Eventually, we may reach the minority spin band edge, at which the 


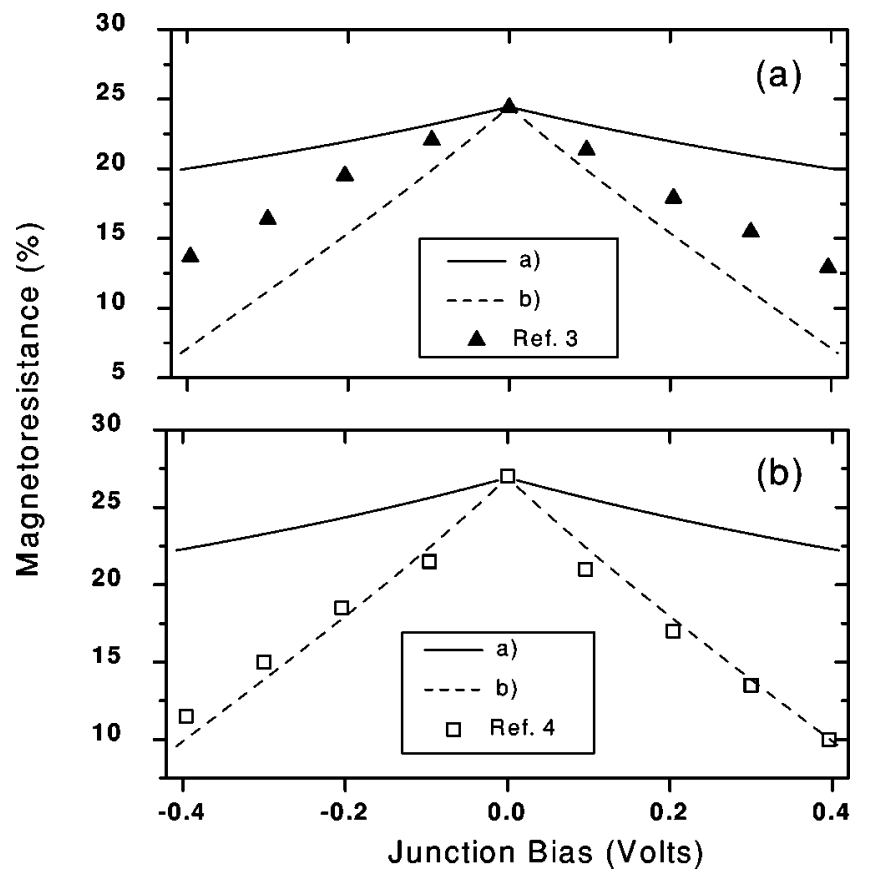

FIG. 2. Magnetoresistance, as defined in Ref. 3, for cases (a) and (b) in Fig. 1. The densities of states are adjusted to the zero-bias value of the MR of the experimental results, which are taken from Ref. 3 in Fig. 2(a) and from Ref. 4 in Fig. 2(b).

density of states vanishes, yielding $R_{\mathrm{AP}} \rightarrow \infty$. Within this limit, the system behaves as an insulator.

In our theory, the MR is just a fingerprint of variations of the electronic density of states with voltage for both spin bands. This result comes about when one assumes factorization (2), implying that $\Delta R / R$ is almost independent of the tunneling mechanism (insensitive to details of the tunneling barrier), (being just) a density of states effect. This fact may be tested experimentally if one is able to prepare a family of "good" tunneling junctions with the same magnetic electrodes but changes some parameters of the insulating barrier (for instance, the barrier width). As expected, the resistance ( $\mathrm{P}$ or AP) will change, but our prediction is that the MR will behave as a universal function of the voltage, and will have the same value as the absolute MR. Deviation from this value may be used as a criterion for the quality of the junction, and may indicate the presence of barrier shorts or pinholes. ${ }^{14}$ It was also suggested in Ref. 15 to locally modify the density of states at the tunneling interface in a controlled manner in order to monitor the tunnel resistance and MR.

Temperature $(T)$ effects can also be taken into account through relation (1) with the broadening of the Fermi distributions, but a rough estimate shows that the effect should be similar to that of applied voltage of $e V \approx 2 k_{B} T$, with effective lowering of the barrier height, smaller resistance, and softening of the zero-bias anomaly, in agreement with experiments.

The following conclusions are pertinent: (i) the overall variation of the tunneling current with voltage can be explained by elastic tunneling for spin conserving currents. We propose that this variation is just a density of states effect. Inelastic scattering is not needed to explain the experiments. In any case, the large variations shown in the present calcu- lation have to be considered in any alternative model; (ii) for the data in Refs. 3 and 4, the anomalies in the currents and the magnetoresistances can be explained within this simple framework, provided that the ratio of majority to minority spin electrons is of the order of 2.2-2.5, at the Fermi level. If one is allowed to choose an appropriate configuration of the bands (see Fig. 1), a maximum, a minimum or a mix of both can appear at the anomaly (as was observed in Ref. 8); (iii) the results may be sample dependent, since oxidation states inside the metal, at the interface, and in the oxide layer may contribute to polarization of the current, thereby inducing local changes in the densities of states. Alternatively, it may also, happen like suggested in Refs. 11, 16, and 17, that the current has contributions from conduction paths (pinholes) that provide large values of magnetoresistance ${ }^{18}$ due to domain wall scattering. ${ }^{19}$ In this last case, the contribution from $d$ electrons may be important, and the density of states will have mixed contributions from $s$ and $d$ electrons, and a variety of topologies in the $\mathrm{MR} .^{20}$

This work was supported by EU Project No. IST-200026011. One of the authors is a visiting scientist at the Laboratorio de Física de Sistemas Pequeños y Nanotecnología, Consejo Superior de Investigaciones Científicas (CSIC), Madrid, Spain. He is grateful to the LFSPN for hospitality and support. He also acknowledges partial financial support from Fundação de Amparo à Pesquisa do Estado de São Paulo (Brazil) through project No. FAPESP 2000/09434-3.

${ }^{1}$ M. Julliere, Phys. Lett. 54A, 225 (1975).

${ }^{2}$ T. Miyazaki and N. Tezuka, J. Magn. Magn. Mater. 139, L231 (1995); J. S. Moodera, L. R. Kinder, T. M. Wong, and R. Meservey, Phys. Rev. Lett. 74, 3273 (1995).

${ }^{3}$ S. Zhang, P. M. Levy, A. C. Marley, and S. S. P. Parkin, Phys. Rev. Lett. 79, 3744 (1997)

${ }^{4}$ J. S. Moodera, J. Nowak, and R. J. M. van de Veerdonk, Phys. Rev. Lett. 80, 2941 (1998)

${ }^{5}$ J. G. Simmons, J. Appl. Phys. 34, 1793 (1963); J. Phys. D 4, 613 (1971).

${ }^{6}$ Y. Ando, J. Murai, H. Kubota, and T. Miyazaki, J. Appl. Phys. 87, 5209 (2000).

${ }^{7}$ U. Bänninger, G. Busch, M. Campagna, and H. C. Siegmann, Phys. Rev. Lett. 25, 585 (1970).

${ }^{8}$ M. Sharma, S. X. Wang, and J. H. Nickel, Phys. Rev. Lett. 82, 616 (1999).

${ }^{9}$ V. L. Moruzzi, J. F. Janak, and A. R. Williams, Calculated Electronic Properties of Metals (Pergamon, New York, 1978).

${ }^{10}$ J. A. Hertz and K. Aoi, Phys. Rev. B 8, 3252 (1973).

${ }^{11}$ N. García, Appl. Phys. Lett. 77, 1351 (2000).

${ }^{12}$ R. Meservey and P. M. Tedrow, Phys. Rep. 238, 173 (1994).

${ }^{13}$ It is interesting to note that the MR found in Ref. 4 is symmetrical in relation to the polarity of voltage, in spite of the fact that the resistances are highly asymmetric [therefore the data were not shown in Fig. 1(b)]. The MR does not show either any indication of the magnon peaks found with IETS at small bias (after Ref. 4).

${ }^{14}$ J. J. Ȧkerman, J. M. Slaughter, R. W. Dave, and I. K. Schuller, Appl. Phys. Lett. 79, 3104 (2001).

${ }^{15}$ P. LeClair, J. T. Kohlhepp, H. J. M. Swagten, and W. J. M. de Jonge, Phys. Rev. Lett. 86, 1066 (2001).

${ }^{16}$ C. L. Platt, A. S. Katz, R. C. Dynes, and A. E. Berkowitz, Appl. Phys. Lett. 75, 127 (1999)

${ }^{17}$ B. J. Jönsson-Äkerman, R. Escudero, C. Leighton, S. Kim, and I. K. Schuller, and D. A. Rabson, Appl. Phys. Lett. 77, 1870 (2000).

${ }^{18}$ N. García, M. Muñoz, and Y.-W. Zhao, Phys. Rev. Lett. 82, 2923 (1999).

${ }^{19}$ G. G. Cabrera and L. M. Falicov, Phys. Status Solidi B 61, 539 (1974); G. Tatara, Y.-W. Zhao, M. Muñoz, and N. García, Phys. Rev. Lett. 83, 2030 (1999)

${ }^{20}$ N. García, H. Rohrer, I. G. Saveliev, and Y.-W. Zhao, Phys. Rev. Lett. 85, 2030 (2000) 